\title{
REDUCED LAGRANGIANS FOR FIELD THEORIES WITH NON-LINEAR COUPLING
}

\author{
V. Ye. Shpytko ${ }^{1 *}$, J. W. Darewych ${ }^{2}$ \\ ${ }^{1}$ Institute for Condensed Matter Physics of NAS of Ukraine, \\ 1 Svientsitskii Str., Lviv, UA-79011, Ukraine \\ ${ }^{2}$ Department of Physics and Astronomy, York University, \\ Toronto, Ontario, M3J 1P3, Canada
}

(Received May 25, 2002)

\begin{abstract}
We consider a reformulation of various models in QFT in which field equations are used to express the mediating fields in terms of matter fields. Scalar QED and SU(2) gauge fields are considered in particular. We show that for these cases the interaction term of the reformulated Lagrangian can be expressed in a series of terms, which contain matter fields and the Green functions of the mediating fields only.

Key words: quantum field theory, classical field theory, scalar electrodynamics, gauge field, effective Lagrangian.

PACS number(s): 03.50.-z, 03.50.Kk, 11.10.Lm, 11.15.-q
\end{abstract}

\section{INTRODUCTION}

The description of relativistic bound states in QFT continues to be a challenging problem, particularly for strongly coupled systems. The traditional method of treating relativistic bound states within QFT is by means of the Bethe-Salpeter (BS) equation [1]. However, this approach has its limitations, among them the existence of relative-time coordinates, difficulty of implementation for systems of more than two bodies, and the (in practice) perturbative treatment of interactions.

Among various alternatives to the BS equation, such as the quasi-potential approach [2] or infinite-component wave equations [3], the approach which incorporates the ideas of direct interaction theory [4] into QFT commends itself as an effective and comparatively simple method for treating bound state problems [5]. This approach involves the following steps:

i. Elimination of the degrees of freedom of the mediating fields and the consequent construction of an equivalent non-local Lagrangian for the system.

ii. Construction of the corresponding Hamiltonian of the QFT and its quantization.

iii. Derivation and solution of the resulting few-body equations.

This approach was applied to the two-scalar [6], twofermion [7] and scalar-fermion [8] problems in models with scalar mediating fields (Yukawa coupling), as well as to the two-fermion problem in quantum electrodynamics (QED) [9].

The starting point of this approach is the Lagrangian function for the "matter" fields (scalar or fermionic) and the massless (or massive) "mediating" fields. The corresponding classical (Euler-Lagrange) field equations are used to express the mediating fields in terms of the matter fields by means of Green's functions. This solution is then used to construct an effective reduced Lagrangian which is non-local but depends on matter fields only. The structure of the effective action is similar to the Fokkertype action integrals in relativistic direct interaction theory [4].

The construction of the reduced Lagrangians is easily realizable in a few cases, such as those listed above [6-9]. But, in general, the elimination of mediating fields cannot be done exactly in closed form. One example is scalar electrodynamics, in which spinless particles represented by scalar "matter fields" $\varphi_{a}(a=1,2)$ interact via electromagnetic (massless vector) fields $A^{\mu}$. The mediating-field equations are just the Maxwell equations

$$
\partial_{\nu} F^{\nu \mu}=\partial_{\nu} \partial^{\nu} A^{\mu}-\partial^{\mu}\left(\partial^{\nu} A_{\nu}\right)=J^{\mu},
$$

with the source current

$$
J^{\mu}=-\sum_{a=1}^{2}\left[i e\left(\varphi_{a} \partial^{\mu} \varphi_{a}^{*}-\varphi_{a}^{*} \partial^{\mu} \varphi_{a}\right)+2 e^{2} \varphi_{a}^{*} \varphi_{a} A^{\mu}\right]
$$

Since the source current contains both matter and mediating fields, we cannot solve these equations for the mediating electromagnetic field in terms of the matter fields using traditional Green function methods.

Therefore, we need to generalize the approach used for the solvable cases described above [6-9], but in a way that will avoid approximations as much as possible, since the subsequent steps (such as the construction of the Hamiltonian, quantization, etc.) may depend on the approximation scheme, which, in turn, may undermine the rigorous nature of the method. Thus, it is important to have a reformulation of the theory, which is free of 


\section{YE. SHPYTKO, J. W. DAREWYCH}

approximation schemes, at least at the classical level.

In the present paper we construct an implicit form of the reduced Lagrangian, which can be used for some physically interesting cases in either the canonical Hamiltonian or path-integral formulation. In this way approximations can be avoided at the classical level, and can be applied as necessary at the quantized level. We shall construct the implicit form of the reduced Lagrangian for the following cases:

1. Complex scalar matter fields interacting via a massless scalar field (scalar coupling);

2. Complex scalar matter fields interacting via the electromagnetic field (vector coupling);

3. Scalar iso-triplet matter fields interacting via SU(2)-gauge field (SU(2)-gauge coupling).

For case 1 the scalar coupling is of the "minimal" type (see [10]), not of the Yukawa-type (as in Ref. [6]). This model will illustrate how the difficulty in applying the simple elimination procedure used for the Yukawa-type coupling can be circumvented.

\section{SCALAR COUPLING}

\section{A. Lagrangian density and field equations}

The Lagrangian density of two complex scalar fields $\varphi_{a}(x)$ with masses $m_{a}(a=1,2)$ interacting via a scalar real massless field $\chi(x)$, with scalar coupling, is

$$
\begin{aligned}
L & =\sum_{a=1}^{2}\left[\partial_{\mu} \varphi_{a}^{*} \partial^{\mu} \varphi_{a}-\left(m_{a}+\alpha \chi\right)^{2} \varphi_{a}^{*} \varphi_{a}\right] \\
& +\frac{1}{2} \partial_{\mu} \chi \partial^{\mu} \chi
\end{aligned}
$$

where $\alpha$ is a dimensionless coupling constant. The corresponding Euler-Lagrange equations are

$$
\begin{gathered}
\left(\square+\left(m_{a}+\alpha \chi\right)^{2}\right) \varphi_{a}=0, \\
\square \chi=-\alpha\left(\rho_{0}+\rho_{1} \chi\right),
\end{gathered}
$$

where $\square=\partial_{\mu} \partial^{\mu}$, and

$$
\rho_{0}=2 \sum_{a=1}^{2} m_{a} \varphi_{a}^{*} \varphi_{a}, \quad \rho_{1}=2 \alpha \sum_{a=1}^{2} \varphi_{a}^{*} \varphi_{a}
$$

Equation (2.3) for the $\chi$ field, but with $\rho_{1}=0$ on the r.h.s., is the same as that for the scalar Yukawa model [6]. In that case one can find the solution using the standard Green function method, namely

$$
\chi=\chi_{0}-\alpha\left\langle D * \rho_{0}\right\rangle
$$

where $\chi_{0}$ is a solution of the homogeneous equation, $\square \chi_{0}=0$, and

$$
\left\langle D * \rho_{0}\right\rangle=\square^{-1} \rho_{0}=\int d x^{\prime} D\left(x-x^{\prime}\right) \rho_{0}\left(x^{\prime}\right)
$$

where $d x^{\prime}=d t^{\prime} d \mathbf{r}^{\prime}$, and $D\left(x-x^{\prime}\right)$ is a covariant Green function (or chion propagator, in the language of QFT):

$$
\square D\left(x-x^{\prime}\right)=\delta^{4}\left(x-x^{\prime}\right)
$$

However, in the present case, with $\rho_{1} \neq 0$, this simple procedure does not work.

From now on we shall put $\chi_{0}=0$, i.e., we will neglect the free $\chi$-field. This means that we shall not consider phenomena that involve annihilation or decay with the emission of free chions, but only stable bound states (or elastic scattering). We seek a solution for the $\chi$-field in the form

$$
\chi=\langle D * \bar{\rho}\rangle
$$

where $\bar{\rho}$ is a functional of the matter fields $\varphi_{a}, \varphi_{a}^{*}$. Substitution of (2.8) into (2.3) gives the integral equation

$$
\bar{\rho}=-\alpha\left(\rho_{0}+\rho_{1}\langle D * \bar{\rho}\rangle\right)
$$

which specifies the functional $\bar{\rho}$. Let us write the solution of (2.9) in the form

$$
\bar{\rho}=-\alpha\left(1+\alpha \rho_{1}\langle D * \ldots\rangle\right)^{-1} \rho_{0}
$$

This formula contains the expression $(1+\alpha\langle D * \ldots\rangle)^{-1}$, which is to be understood as an operator inverse. We note that this construction looks like the fraction $1 /(1+x)$ and can be treated as an algebraic expression but in terms of operator algebra. We recall that $1 /(1+x)$ can be represented by convergent series for the two cases, $x<1$ and $x>1$ :

$$
\begin{aligned}
& \frac{1}{1+x} \stackrel{x \leqq 1}{=} \sum_{k=0}^{\infty}(-x)^{k}, \\
& \frac{1}{1+x} \stackrel{x \geq 1}{=}-\sum_{k=1}^{\infty}\left(-\frac{1}{x}\right)^{k} .
\end{aligned}
$$

Analogously, we can express the functional $\bar{\rho}(2.10)$ as follows:

$$
\begin{aligned}
& \bar{\rho} \stackrel{\alpha \leq 1}{=}-\alpha \sum_{k=0}^{\infty}\left(-\alpha \rho_{1}\langle D * \ldots\rangle\right)^{k} \rho_{0}, \\
& \bar{\rho} \stackrel{\alpha \geq 1}{=} \alpha \sum_{k=1}^{\infty}\left(-\frac{1}{\alpha} \square \frac{1}{\rho_{1}}\right)^{k} \rho_{0} .
\end{aligned}
$$


The notation $\stackrel{\alpha \leq 1}{=}$ (or $\stackrel{\alpha \geq 1}{=}$ ) indicates that the representation (2.13) (or (2.14)) is appropriate for the case of the weak (or strong) coupling, i. e. for $\alpha \ll 1$ (or $\alpha \gg 1$ ). Both these representations are well-defined in terms of operator algebra. Moreover, it is easy to demonstrate that both of these formally constructed solutions satisfy Eq. (2.9):

$$
\begin{aligned}
\bar{\rho} & \underline{\alpha}=1 \\
= & \alpha \sum_{k=0}^{\infty}\left[-\alpha \rho_{1}\langle D * \ldots\rangle\right]^{k} \rho_{0}=-\alpha \rho_{0}-\alpha \sum_{k=1}^{\infty}\left[-\alpha \rho_{1}\langle D * \ldots\rangle\right]^{k} \rho_{0} \\
& =-\alpha \rho_{0}+\alpha \sum_{k=1}^{\infty} \alpha \rho_{1}\left\langle D *\left[-\alpha \rho_{1}\langle D * \ldots\rangle\right]^{k-1} \rho_{0}\right\rangle=-\alpha \rho_{0}+\alpha^{2} \rho_{1} \\
& \times\left\langle D * \sum_{k=0}^{\infty}\left[-\alpha \rho_{1}\langle D * \ldots\rangle\right]^{k} \rho_{0}\right\rangle=-\alpha \rho_{0}-\alpha \rho_{1}\langle D * \bar{\rho}\rangle, \\
\bar{\rho} & \stackrel{\alpha \geq 1}{=} \sum_{k=1}^{\infty}\left(-\frac{1}{\alpha} \square \frac{1}{\rho_{1}}\right)^{k} \rho_{0}=\alpha\left(\sum_{k=0}^{\infty}\left(-\frac{1}{\alpha} \square \frac{1}{\rho_{1}}\right)^{k}-1\right) \rho_{0} \\
& =\alpha\left(\sum_{k=1}^{\infty}\left(-\frac{1}{\alpha} \square \frac{1}{\rho_{1}}\right)^{k-1}-1\right) \rho_{0}=\left(-\frac{1}{\alpha} \square \frac{1}{\rho_{1}}\right)^{-1} \\
& \times \alpha \sum_{k=1}^{\infty}\left(-\frac{1}{\alpha} \square \frac{1}{\rho_{1}}\right)^{k} \rho_{0}-\alpha \rho_{0}=-\alpha \rho_{0}-\alpha \rho_{1}\langle D * \bar{\rho}\rangle,
\end{aligned}
$$

where we have used the identity

$$
\left(-\frac{1}{\alpha} \square \frac{1}{\rho_{1}}\right)^{-1}=-\alpha \rho_{1}\langle D * \ldots\rangle .
$$

Thus, we have indeed constructed representations for the functional $\bar{\rho}(2.8)$.

Expressions (2.13) and (2.14), together with Eq. (2.8), allow us to express the mediating field $\chi$ in terms of the particle fields and the Green function for the mediating field, but in the form of a series. In particular, representation (2.11) corresponds to a perturbative solution $\chi=\alpha \chi_{1}+\alpha^{2} \chi_{2}+\alpha^{3} \chi_{3}+\ldots$ of the field equation (2.3), where

$$
\begin{aligned}
& \chi_{1}=-\left\langle D * \rho_{0}\right\rangle, \quad \chi_{2}=\left\langle D * \rho_{1}\left\langle D * \rho_{0}\right\rangle\right\rangle, \\
& \chi_{3}=-\left\langle D * \rho_{1}\left\langle D * \rho_{1}\left\langle D * \rho_{0}\right\rangle\right\rangle\right\rangle, \ldots .
\end{aligned}
$$

\section{B. Reduced Lagrangian}

In this subsection we shall consider a reformulation of the theory which will be termed the reduced Lagrangian description. The word reduced, we recall, means that the reformulated Lagrangian will not contain the mediating field $\chi$ explicitly.
Let us substitute the formal solution (2.8) into the Lagrangian density (2.1). We are interested in the Hamiltonian least action principle, therefore we shall discard surface terms in the Lagrangian density, since they have no influence on the equations of motion. Accordingly, we

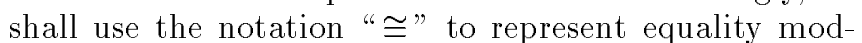
ulo surface terms. For example, the last term in the Lagrangian density (2.1) can be presented as follows:

$$
\begin{aligned}
\frac{1}{2} \partial_{\mu} \chi \partial^{\mu} \chi & =\frac{1}{2} \partial_{\mu}\left(\chi \partial^{\mu} \chi\right)-\frac{1}{2} \chi \partial_{\mu} \partial^{\mu} \chi \\
& \simeq-\frac{1}{2} \chi \partial_{\mu} \partial^{\mu} \chi=-\frac{1}{2} \bar{\rho}\langle D * \bar{\rho}\rangle
\end{aligned}
$$

Using this result and Eq. (2.9), the Lagrangian density (2.1) can be written in the form

$$
L=\sum_{a=1}^{2}\left[\partial_{\mu} \varphi_{a}^{*} \partial^{\mu} \varphi_{a}-m_{a}^{2} \varphi_{a}^{*} \varphi_{a}\right]-\frac{\alpha}{2} \rho_{0}\langle D * \bar{\rho}\rangle
$$

We show next that the Lagrangian density (2.20) gives the field equations (2.2), where the expression for the mediating field $\chi$ is given by (2.8). To do so we need only the functional for $\bar{\rho}$, defined by equation (2.9). Variation of Eq. (2.9) gives the result 


$$
\delta \bar{\rho}=-\alpha\left(\delta \rho_{0}+\delta \rho_{1}\langle D * \bar{\rho}\rangle+\rho_{1}\langle D * \delta \bar{\rho}\rangle\right) .
$$

Then the variation of the action with the Lagrangian density (2.20) gives

$$
\delta S=\delta S_{\text {free }}+\delta S_{\mathrm{int}}=\int d x \delta L \cong \int d x\left(\sum_{b=1}^{2} \delta \varphi_{b}^{*}\left[-\square-m_{b}^{2}\right] \varphi_{b}+(\text { c.c. })+\delta L_{\mathrm{int}}\right),
$$

where (c.c.) denotes complex conjugate terms, and

$$
\begin{aligned}
\delta S_{\mathrm{int}} & =\int d x \delta L_{\mathrm{int}}=-\frac{\alpha}{2} \int d x\left(\delta \rho_{0}\langle D * \bar{\rho}\rangle+\rho_{0}\langle D * \delta \bar{\rho}\rangle\right) \\
& =\frac{1}{2} \int d x\left(\langle D * \bar{\rho}\rangle\left(\delta \bar{\rho}+\alpha \delta \rho_{1}\langle D * \bar{\rho}\rangle+\alpha \rho_{1}\langle D * \delta \bar{\rho}\rangle\right)+\langle D * \delta \bar{\rho}\rangle\left(\bar{\rho}+\alpha \rho_{1}\langle D * \bar{\rho}\rangle\right)\right) \\
& =\int d x\langle D * \bar{\rho}\rangle\left(\delta \bar{\rho}+\frac{\alpha}{2} \delta \rho_{1}\langle D * \bar{\rho}\rangle+\alpha \rho_{1}\langle D * \delta \bar{\rho}\rangle\right)=-\int d x\langle D * \bar{\rho}\rangle\left(\alpha \delta \rho_{0}+\frac{\alpha}{2} \delta \rho_{1}\langle D * \bar{\rho}\rangle\right) .
\end{aligned}
$$

We have here used equations (2.9) and (2.21), and the fact that we have chosen the Green function to be symmetric: $D\left(x-x^{\prime}\right)=D\left(x^{\prime}-x\right)$. Taking into account Eqs. (2.22), (2.23) and the definitions (2.4) we obtain the field equations

$$
\left(\square+\left(m_{a}+\langle D * \bar{\rho}\rangle\right)^{2}\right) \varphi_{a}=0,
$$

and the corresponding complex conjugate equations.

\section{SCALAR QED AND VECTOR COUPLING}

The Lagrangian density of two complex scalar fields, $\varphi_{1}(x)$ and $\varphi_{2}(x)$, interacting electromagnetically, is

$$
L=\sum_{a=1}^{2}\left[\left(D_{\mu} \varphi_{a}\right)^{*}\left(D^{\mu} \varphi_{a}\right)-m_{a}^{2} \varphi_{a}^{*} \varphi_{a}\right]-\frac{1}{4} F^{\mu \nu} F_{\mu \nu}
$$

or

$$
\begin{aligned}
L & \cong \sum_{a=1}^{2}\left[\left(D_{\mu} \varphi_{a}\right)^{*}\left(D^{\mu} \varphi_{a}\right)-m_{a}^{2} \varphi_{a}^{*} \varphi_{a}\right]+\frac{1}{2} A_{\nu}\left(g^{\nu \mu} \square-\partial^{\nu} \partial^{\mu}\right) A_{\mu} \\
& =\sum_{a=1}^{2}\left[\partial_{\mu} \varphi_{a}^{*} \partial^{\mu} \varphi_{a}-m_{a}^{2} \varphi_{a}^{*} \varphi_{a}\right]+e A^{\mu} \stackrel{0}{J}_{\mu}+\frac{e}{2} \rho A_{\mu} A^{\mu}+\frac{1}{2} A_{\nu}\left(g^{\nu \mu} \square-\partial^{\nu} \partial^{\mu}\right) A_{\mu},
\end{aligned}
$$

where $g^{\nu \mu}$ is the Minkowski metric tensor, and

$$
\begin{gathered}
\left(D_{\mu} \varphi\right)^{*}=\left(\partial_{\mu}-i e A_{\mu}\right) \varphi^{*}, \quad D_{\mu} \varphi=\left(\partial_{\mu}+i e A_{\mu}\right) \varphi, \quad F_{\mu \nu}=\partial_{\mu} A_{\nu}-\partial_{\nu} A_{\mu}, \\
\stackrel{J}{J}_{\mu}=i \sum_{a=1}^{2}\left(\varphi_{a} \partial \varphi_{a}^{*}-\varphi_{a}^{*} \partial \varphi_{a}\right), \quad \rho=2 e \sum_{a=1}^{2} \varphi_{a} \varphi_{a}^{*} .
\end{gathered}
$$


The corresponding Euler-Lagrange equations are the coupled Klein-Gordon-Maxwell equations:

$$
\left(D_{\mu} D^{\mu}+m_{a}^{2}\right) \varphi_{a}=0
$$

and Eq. (1.1) with the conserved current

$$
\begin{aligned}
& \partial_{\mu} J^{\mu}=0, \\
& J^{\mu}=-e\left(\stackrel{0}{J}^{\mu}+\rho A^{\mu}\right),
\end{aligned}
$$

c.f. Eq. (1.2). The operator $g^{\nu \mu} \square-\partial^{\nu} \partial^{\mu}$ is degenerate and therefore has no inverse. Thus, we have to choose some gauge. It is convenient to use the Lorentz gauge:

$$
\partial_{\mu} A^{\mu}=0
$$

We can now use the approach of Section II to eliminate the electromagnetic field. Let us put

$$
A^{\mu}=\left\langle D * \bar{J}^{\mu}\right\rangle
$$

where $D$ is a symmetric Green function (c.f. Eq. (2.7)) and $\bar{J}^{\mu}$ is a functional of the matter fields $\varphi_{a}, \varphi_{a}^{*}$. Substitution of (3.9) into (1.1) (in Lorentz gauge) gives

$$
\bar{J}^{\mu}=-e\left(\stackrel{0}{J}^{\mu}+\rho\left\langle D * \bar{J}^{\mu}\right\rangle\right)
$$

which is the defining equation for $\bar{J}_{\mu}$. It is evident from (3.10) and (3.9) that $\bar{J}^{\mu}$ is equal to the current (3.7) (or (1.2)), except that it is expressed in terms of the matter fields only. The formal solution of (3.10) can be expressed as

$$
\bar{J}_{\mu}=-\frac{e}{1+e \rho\langle D * \ldots\rangle} \stackrel{0}{J}_{\mu}
$$

Again, we can represent the functional $\bar{J}_{\mu}$ in the form of series. In full analogy with the case of scalar coupling (see Eqs. (2.11), (2.12) and (2.13), (2.14)) we have two representations for $\bar{J}_{\mu}$ :

$$
\begin{aligned}
& \bar{J}_{\mu} \stackrel{e \leq 1}{=}-e \sum_{k=0}^{\infty}(-e \rho\langle D * \ldots\rangle)^{k} \stackrel{0}{J}_{\mu}, \\
& \bar{J}_{\mu} \stackrel{e \geq 1}{=} e \sum_{k=1}^{\infty}\left(-\frac{1}{e} \square \frac{1}{\rho}\right)^{k} \stackrel{0}{J}_{\mu} .
\end{aligned}
$$

Representation (3.12) corresponds to the perturbative solution of (1.1),

$$
A_{\mu}=e \stackrel{1}{A} \mu+e^{2} \stackrel{2}{A}_{\mu}+e^{3} \stackrel{3}{A}_{\mu}+\ldots
$$

where

$$
\begin{aligned}
& \stackrel{1}{A}_{\mu}=-\left\langle D * \stackrel{0}{J}_{\mu}\right\rangle, \quad \stackrel{2}{A}_{\mu}=\left\langle D * \rho\left\langle D * \stackrel{0}{J}_{\mu}\right\rangle\right\rangle, \\
& \stackrel{0}{A}_{\mu}=-\left\langle D * \rho\left\langle D * \rho\left\langle D * \stackrel{0}{J}_{\mu}\right\rangle\right\rangle\right\rangle, \ldots
\end{aligned}
$$

Substitution of (3.9) into (3.2) leads to the following reduced Lagrangian density

$$
L=\sum_{\mathrm{a}=1}^{2}\left[\partial_{\mu} \varphi_{a}^{*} \partial^{\mu} \varphi_{a}-m_{a}^{2} \varphi_{a}^{*} \varphi_{a}\right]+\frac{e}{2} \stackrel{0}{J}_{\mu}\left\langle D * \bar{J}^{\mu}\right\rangle
$$

which is the basis of the variational principle in our approach.

To deduce the field equations we vary both sides of Eq. (3.10),

$$
\delta \bar{J}^{\mu}=-e\left(\delta \stackrel{0}{J}^{\mu}+\delta \rho\left\langle D * \bar{J}^{\mu}\right\rangle+\rho\left\langle D * \delta \bar{J}^{\mu}\right\rangle\right),
$$

and thence calculate the variation of the interaction part of the action:

$$
\begin{aligned}
\int d x \delta L_{\mathrm{int}} & =\frac{e}{2} \int d x\left[\delta \stackrel{0}{J}_{\mu}\left\langle D * \bar{J}^{\mu}\right\rangle+\stackrel{0}{J}_{\mu}\left\langle D * \delta \bar{J}^{\mu}\right\rangle\right] \\
& =\frac{e}{2} \int d x\left[\left\langle D * \bar{J}_{\mu}\right\rangle\left(-\delta \bar{J}^{\mu}-e\left(\delta \rho\left\langle D * \bar{J}^{\mu}\right\rangle+\rho\left\langle D * \bar{J}^{\mu}\right\rangle\right)\right)-\left\langle D * \delta \bar{J}_{\mu}\right\rangle\left(\bar{J}^{\mu}+e \rho\left\langle D * \bar{J}^{\mu}\right\rangle\right)\right] \\
& =-\int d x\left\langle D * \bar{J}_{\mu}\right\rangle\left(\delta \bar{J}^{\mu}+\frac{e}{2} \delta \rho\left\langle D * \bar{J}^{\mu}\right\rangle+e \rho\left\langle D * \delta \bar{J}^{\mu}\right\rangle\right)=e \int d x\left\langle D * \bar{J}_{\mu}\right\rangle\left(\delta \bar{J}^{\mu}+\frac{1}{2} \delta \rho\left\langle D * \bar{J}^{\mu}\right\rangle\right) \\
& \cong e \int d x\left[\sum_{b=1}^{2} \delta \varphi_{b}^{*}\left(-i\left(\varphi_{b} \partial_{\mu}\left\langle D * \bar{J}^{\mu}\right\rangle+2\left\langle D * \bar{J}^{\mu}\right\rangle \partial_{\mu} \varphi_{b}\right)+e \varphi_{b}\left\langle D * \bar{J}^{\mu}\right\rangle^{2}\right)+(\text { c.c. })\right]
\end{aligned}
$$

As a result we obtain the following equations:

$$
\left(\bar{D}_{\mu} \bar{D}^{\mu}+m_{a}^{2}\right) \varphi_{a}=0
$$




\section{YE. SHPYTKO, J. W. DAREWYCH}

and the complex conjugate

$$
\left(\left(\bar{D}_{\mu} \bar{D}^{\mu}\right)^{*}+m_{a}^{2}\right) \varphi_{a}^{*}=0
$$

where

$$
\bar{D}_{\mu}=\partial_{\mu}+i e\left\langle D * \bar{J}_{\mu}\right\rangle
$$

It is easy to verify that

$$
\partial_{\mu} \bar{J}^{\mu}=0
$$

follows from equations (3.19) and (3.20). This current conservation law, in turn, implies the Lorentz gauge:

$$
\partial_{\mu} A^{\mu}=\partial_{\mu}\left\langle D * \bar{J}^{\mu}\right\rangle=0
$$

Thus, we have transformed the original "local" description, based on the Lagrangian density (3.1) with its explicit dependence on the mediating electromagnetic field, into an equivalent "non-local" description, based on the Lagrangian density (3.16), which has no explicit dependence on the electromagnetic degrees of freedom.

Note that we did not need an explicit form of the functional $\bar{J}_{\mu}$ to construct the non-local Lagrangian density (3.16). We used only the integral equation (3.10) for $\bar{J}_{\mu}$. Therefore, we expect that the same method will work in the case of non-Abelian gauge fields.

\section{SU(2)-GAUGE FIELDS}

\section{A. Lagrangian density and field equations}

In this section we construct the reduced Lagrangian for the case of "matter" fields interacting via non-Abelian gauge fields. We start with the simple model defined by the SU(2) gauge-invariant Lagrangian density

$$
\begin{aligned}
L & =\frac{1}{2} \sum_{a=1}^{2}\left(D_{\mu} \boldsymbol{\varphi}_{a} \cdot D^{\mu} \boldsymbol{\varphi}_{a}-m_{a}^{2} \boldsymbol{\varphi}_{a} \cdot \boldsymbol{\varphi}_{a}\right) \\
& -\frac{1}{4} \boldsymbol{F}_{\mu \nu} \cdot \boldsymbol{F}^{\mu \nu}
\end{aligned}
$$

where each field $\boldsymbol{\varphi}_{a}(a=1,2)$ has three components $\boldsymbol{\varphi}=\left(\varphi_{1}, \varphi_{2}, \varphi_{3}\right)$, i.e., it belongs to the vector representation of $\mathrm{SU}(2)$ and describes particles with isospin and masses $m_{a}$. The symbol $D_{\mu}$ denotes the covariant derivative:

$$
D_{\mu} \boldsymbol{\varphi}=\partial_{\mu} \boldsymbol{\varphi}+g \boldsymbol{A}_{\mu} \times \boldsymbol{\varphi}
$$

and

$$
\boldsymbol{F}_{\mu \nu}=\partial_{\mu} \boldsymbol{A}_{\nu}-\partial_{\nu} \boldsymbol{A}_{\mu}+g \boldsymbol{A}_{\mu} \times \boldsymbol{A}_{\nu}
$$

The corresponding equations for the gauge fields are

$$
D_{\mu} \boldsymbol{F}^{\mu \nu}=g \sum_{\mathrm{a}=1}^{2} D^{\nu} \boldsymbol{\varphi}_{a} \times \boldsymbol{\varphi}_{a}
$$

We rewrite them in a form more convenient for further use:

$$
\begin{aligned}
\square \boldsymbol{A}^{\nu} & -\partial^{\mu} \partial^{\nu} \boldsymbol{A}_{\mu}+g \partial_{\mu}\left(\boldsymbol{A}^{\mu} \times \boldsymbol{A}^{\nu}\right)+g \boldsymbol{A}_{\mu} \\
& \times\left(\partial^{\mu} \boldsymbol{A}^{\nu}-\partial^{\nu} \boldsymbol{A}^{\mu}\right)+g^{2} \boldsymbol{A}_{\mu} \times\left(\boldsymbol{A}^{\mu} \times \boldsymbol{A}^{\nu}\right) \\
& -g^{2} \sum_{a=1}^{2}\left(\boldsymbol{A}^{\nu} \times \boldsymbol{\varphi}_{a}\right) \times \boldsymbol{\varphi}_{a}=g \boldsymbol{J}^{\nu}, \\
\boldsymbol{J}^{\nu} & =\sum_{a=1}^{2} \partial^{\nu} \boldsymbol{\varphi}_{a} \times \boldsymbol{\varphi}_{a} .
\end{aligned}
$$

To find a solution

$$
\tilde{\boldsymbol{A}}_{\nu}=\boldsymbol{A}_{\nu}(\varphi)
$$

of Eq. (4.5), we must choose a gauge. Let us choose the covariant gauge,

$$
D^{\nu} \boldsymbol{A}_{\nu}=\partial^{\nu} \boldsymbol{A}_{\nu}=0
$$

and suppose that we know the solution (4.7) in this gauge. Then $\tilde{\boldsymbol{A}}$ will satisfy the following equation

$$
\begin{gathered}
\square \tilde{\boldsymbol{A}}^{\nu}+g \tilde{\boldsymbol{A}}_{\mu} \times\left(2 \partial^{\mu} \tilde{\boldsymbol{A}}^{\nu}-\partial^{\nu} \tilde{\boldsymbol{A}}^{\mu}\right)+g^{2} \tilde{\boldsymbol{A}}_{\mu} \times\left(\tilde{\boldsymbol{A}}^{\mu} \times \tilde{\boldsymbol{A}}^{\nu}\right) \\
-g^{2} \sum_{\mathrm{a}=1}^{2}\left(\tilde{\boldsymbol{A}}^{\nu} \times \boldsymbol{\varphi}_{a}\right) \times \boldsymbol{\varphi}_{a}=g \tilde{J}^{\nu}
\end{gathered}
$$

The corresponding equations for the matter fields $\boldsymbol{\varphi}_{a}$ have the form

$$
\left(D_{\nu} D^{\nu}+m_{a}^{2}\right) \varphi_{a}=0
$$

\section{B. Reduced Lagrangian}

Substituting the formal solution (4.7) into the Lagrangian density (4.1), and using equation (4.9), we obtain the reduced Lagrangian density

$$
\begin{aligned}
\tilde{L} & =\frac{1}{2} \sum_{a=1}^{2}\left(\partial_{\mu} \boldsymbol{\varphi}_{a} \cdot \partial^{\mu} \boldsymbol{\varphi}_{a}-m_{a}^{2} \boldsymbol{\varphi}_{a} \cdot \boldsymbol{\varphi}_{a}\right) \\
& -\frac{g}{2} \tilde{\boldsymbol{A}}_{\nu} \cdot\left(\stackrel{0}{\nu}^{\nu}+\tilde{\boldsymbol{A}}_{\mu} \times\left(\frac{g}{2} \tilde{\boldsymbol{A}}^{\mu} \times \tilde{\boldsymbol{A}}^{\nu}+\partial^{\mu} \tilde{\boldsymbol{A}}^{\nu}\right)\right)
\end{aligned}
$$




$$
=\frac{1}{2} \sum_{a=1}^{2}\left(\partial_{\mu} \boldsymbol{\varphi}_{a} \cdot \partial^{\mu} \boldsymbol{\varphi}_{a}-m_{a}^{2} \boldsymbol{\varphi}_{a} \cdot \boldsymbol{\varphi}_{a}\right)+\tilde{L}_{\mathrm{int}}
$$

To show that this Lagrangian density gives the field equations (4.10) we proceed as follows. Using (4.9) we obtain

$$
\begin{aligned}
\tilde{\boldsymbol{A}}_{\nu} \cdot \delta \tilde{\boldsymbol{J}}^{\nu} & =\delta \tilde{\boldsymbol{A}}^{\nu} \cdot\left(\square \tilde{\boldsymbol{A}}_{\nu}+2 g\left(\partial_{\nu} \tilde{\boldsymbol{A}}_{\mu}-2 \partial_{\mu} \tilde{\boldsymbol{A}}_{\nu}\right) \times \tilde{\boldsymbol{A}}^{\mu}+3 g^{2} \tilde{\boldsymbol{A}}^{\mu} \times\left(\tilde{\boldsymbol{A}}_{\mu} \times \tilde{\boldsymbol{A}}_{\nu}\right)\right. \\
& \left.-g^{2} \boldsymbol{\varphi}_{a} \times\left(\boldsymbol{\varphi}_{a} \times \tilde{\boldsymbol{A}}_{\nu}\right)\right)-2 g^{2} \delta \boldsymbol{\varphi}_{a} \cdot\left(\tilde{\boldsymbol{A}}_{\nu} \times\left(\tilde{\boldsymbol{A}}^{\nu} \times \boldsymbol{\varphi}_{a}\right)\right) .
\end{aligned}
$$

Moreover,

$$
\delta L_{\mathrm{int}}=-\frac{g}{2}\left[\tilde{\boldsymbol{A}}_{\nu} \cdot \delta \tilde{J}^{\nu}+\delta \tilde{\boldsymbol{A}}_{\nu} \cdot\left(\tilde{J}^{\nu}+2 g \tilde{\boldsymbol{A}}_{\mu} \times\left(\tilde{\boldsymbol{A}}^{\mu} \times \tilde{\boldsymbol{A}}^{\nu}\right)+\tilde{\boldsymbol{A}}_{\mu} \times\left(2 \partial^{\mu} \tilde{\boldsymbol{A}}^{\nu}-\partial^{\nu} \tilde{\boldsymbol{A}}^{\mu}\right)\right)\right] .
$$

Substitution of (4.9), (4.12) into (4.13) gives

$$
\delta L_{\mathrm{int}}=-g \sum_{a=1}^{2} \delta \boldsymbol{\varphi}_{a} \cdot\left(\tilde{\boldsymbol{A}}_{\nu} \times \partial^{\nu} \boldsymbol{\varphi}_{a}+\partial^{\nu}\left(\tilde{\boldsymbol{A}}_{\nu} \times \boldsymbol{\varphi}_{a}\right)+g \tilde{\boldsymbol{A}}^{\nu} \times\left(\tilde{\boldsymbol{A}}_{\nu} \times \boldsymbol{\varphi}_{a}\right)\right) .
$$

As a result we obtain the following equations

$$
\left(\tilde{D}_{\nu} \tilde{D}^{\nu}+m_{a}^{2}\right) \varphi_{a}=0
$$

where

$$
\tilde{D}_{\nu} \varphi=\partial_{\nu} \boldsymbol{\varphi}+g \tilde{\boldsymbol{A}}_{\nu} \times \boldsymbol{\varphi}
$$

After vector multiplication of (4.15) by $\varphi_{a}$ on the right we obtain a covariant conservation law for the matter current:

$$
\sum_{a=1}^{2} \tilde{D}_{\nu}\left(\tilde{D}^{\nu} \boldsymbol{\varphi}_{a} \times \boldsymbol{\varphi}_{a}\right)=0
$$

(in the original description of the system this equation is a direct consequence of the mediator-field equations (4.4)).

We have thus obtained a self-consistent (non-local) La- grangian description, which, in view of (4.7), does not contain the non-Abelian fields explicitly, and which is equivalent to the description based on Lagrangian density (4.1). To derive this result we have used only the property that the functional $\tilde{\boldsymbol{A}}$ is a solution of Eq. (4.9).

However, for the practical calculations it is necessary to find an explicit form of the functional $\tilde{\boldsymbol{A}}$. This cannot be obtained in closed form, given the non-linear nature of Eq. (4.9). Therefore, we propose an approximate perturbative method for obtaining such a representation.

\section{Approximate solution}

Letting

$$
\tilde{\boldsymbol{A}}^{\nu}=\sum_{i=1}^{\infty} g^{i} \boldsymbol{A}^{\nu}
$$

and substituting into (4.9) we arrive at the following chain of equations:

$$
\begin{aligned}
& \square \boldsymbol{A}^{\nu}=0, \\
& \square \stackrel{1}{\boldsymbol{A}^{\nu}}=\stackrel{0}{\boldsymbol{J}^{\nu}}-\stackrel{0}{\boldsymbol{A}_{\mu}} \times \stackrel{0}{\boldsymbol{f}^{\mu \nu}}
\end{aligned}
$$

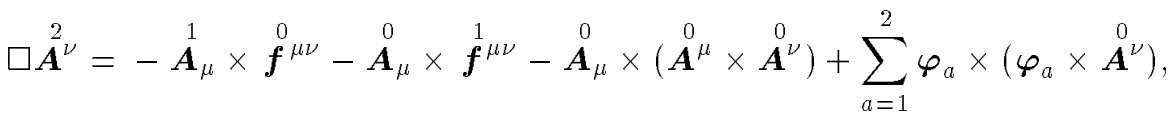




$$
\begin{aligned}
& \square \stackrel{3}{\boldsymbol{A}}^{\nu}=-\stackrel{2}{\boldsymbol{A}_{\mu}} \times \stackrel{0}{\boldsymbol{f}^{\mu \nu}}-\stackrel{0}{\boldsymbol{A}_{\mu}} \times \stackrel{2}{\boldsymbol{f}}^{\mu \nu}-\stackrel{1}{\boldsymbol{A}_{\mu}} \times \stackrel{1}{\boldsymbol{f}^{\mu \nu}}-\stackrel{1}{\boldsymbol{A}_{\mu}} \times\left(\stackrel{0}{\boldsymbol{\boldsymbol { A } ^ { \mu }}} \times \stackrel{0}{\boldsymbol{A}^{\nu}}\right)
\end{aligned}
$$

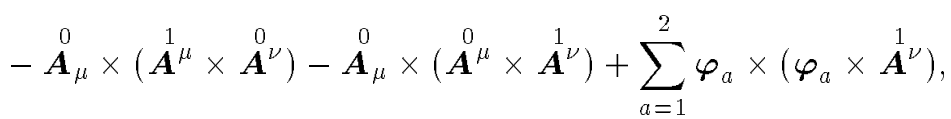

$$
\begin{aligned}
& \square \stackrel{4}{\boldsymbol{A}^{\nu}}=-\stackrel{3}{\boldsymbol{A}} \times \stackrel{0}{\boldsymbol{f}}^{\mu \nu}-\stackrel{0}{\boldsymbol{A}_{\mu}} \times \stackrel{3}{\boldsymbol{f}}^{\mu \nu}-\stackrel{1}{\boldsymbol{A}_{\mu}} \times \stackrel{2}{\boldsymbol{f}}^{\mu \nu}-\stackrel{2}{\boldsymbol{A}_{\mu}} \times \stackrel{1}{\boldsymbol{f}^{\mu \nu}}-\stackrel{2}{\boldsymbol{A}_{\mu}} \times\left(\stackrel{0}{\boldsymbol{A}^{\mu}} \times \stackrel{0}{\boldsymbol{A}^{\nu}}\right) \\
& -\stackrel{0}{\boldsymbol{A}_{\mu}} \times\left(\stackrel{2}{\boldsymbol{A}^{\mu}} \times \stackrel{0}{\boldsymbol{A}^{\nu}}\right)-\stackrel{0}{\boldsymbol{A}_{\mu}} \times\left(\stackrel{0}{\boldsymbol{A}^{\mu}} \times \stackrel{2}{\boldsymbol{A}^{\nu}}\right)-\stackrel{1}{\boldsymbol{A}_{\mu}} \times\left(\stackrel{1}{\boldsymbol{A}^{\mu}} \times \stackrel{0}{\boldsymbol{A}^{\nu}}+\stackrel{0}{\mu} \times \stackrel{1}{\boldsymbol{A}^{\nu}}\right) \\
& -\boldsymbol{A}_{\mu} \times\left(\boldsymbol{A}^{\mu} \times \hat{A}^{\nu}\right)+\sum_{a=1}^{2} \boldsymbol{\varphi}_{a} \times\left(\boldsymbol{\varphi}_{a} \times \stackrel{2}{ }^{\nu}\right), \\
& \square \stackrel{5}{\boldsymbol{A}^{\nu}}=-\stackrel{4}{\boldsymbol{A}_{\mu}} \times \stackrel{0}{\boldsymbol{f}^{\mu \nu}}-\stackrel{0}{\boldsymbol{A}_{\mu}} \times \stackrel{4}{\boldsymbol{f}^{\mu \nu}}-\stackrel{1}{\boldsymbol{A}_{\mu}} \times \stackrel{3}{\boldsymbol{f}^{\mu \nu}}-\stackrel{3}{\boldsymbol{A}_{\mu}} \times \stackrel{1}{\boldsymbol{f}^{\mu \nu}}-\stackrel{2}{\boldsymbol{A}_{\mu}} \times \stackrel{2}{\boldsymbol{f}^{\mu \nu}} \\
& -\stackrel{3}{\boldsymbol{A}_{\mu}} \times\left(\stackrel{0}{\boldsymbol{A}^{\mu}} \times \stackrel{0}{\boldsymbol{A}^{\nu}}\right)-\stackrel{0}{\boldsymbol{A}_{\mu}} \times\left(\stackrel{3}{\boldsymbol{A}^{\mu}} \times \stackrel{0}{\boldsymbol{A}^{\nu}}+\stackrel{0}{\boldsymbol{A}}^{\mu} \times \stackrel{3}{\boldsymbol{A}}^{\nu}\right)
\end{aligned}
$$

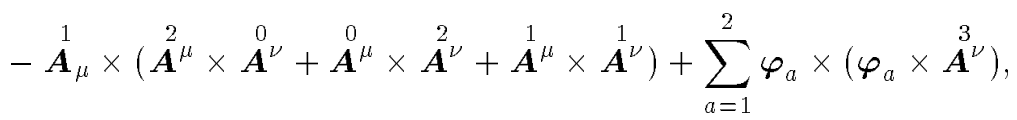

etc., where

$$
\stackrel{i}{\boldsymbol{f}}_{\mu \nu}=2 \partial_{\mu} \stackrel{i}{\boldsymbol{A}}_{\nu}-\partial_{\nu} \stackrel{i}{\boldsymbol{A}_{\mu}}
$$

We assume that there is no radiation corresponding to the free gauge field, that is

$$
\stackrel{2 k}{A}_{\nu}=0, \quad k=0,1,2, \ldots .
$$

As a result we obtain

$$
\tilde{\boldsymbol{A}}_{\nu}=g \sum_{k=0}^{\infty} g^{2 k} \stackrel{2 k+1}{\boldsymbol{A}}_{\nu}
$$

with

$$
\begin{aligned}
& \stackrel{1}{\boldsymbol{A}^{\nu}}=\left\langle D *{\stackrel{0}{\boldsymbol{J}^{\nu}}}^{\nu}\right\rangle \\
& {\stackrel{3}{\boldsymbol{A}^{\nu}}}^{\nu}=-\left\langle D *\left(\hat{A}_{\mu} \times \boldsymbol{f}^{\mu \nu}-\sum_{a=1}^{2} \boldsymbol{\varphi}_{a} \times\left(\boldsymbol{\varphi}_{a} \times{\left.\stackrel{1}{\boldsymbol{A}^{\nu}}\right)}^{\mu}\right)\right\rangle,\right.
\end{aligned}
$$

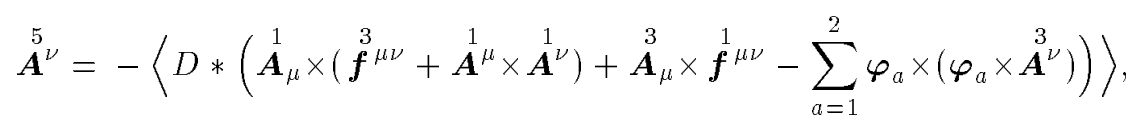

etc., and $\langle D * \ldots\rangle=\square^{-1}$. Using Eqs. (4.28)-(4.30) in (4.27) we obtain the reduced Lagrangian to arbitrary order in $g$ (and, similarly, in (4.11) or (4.16) and (4.15) for the field equations).

The generalization of these results to the case of arbitrary gauge groups is straightforward.

\section{CONCLUDING REMARKS}

We have studied the description of interacting "matter" fields within the "reduced Lagrangian formalism" [5]. In this formalism, the mediating fields are expressed in terms of the corresponding Green functions (propagators) and the matter fields. Such a reformulation, which is convenient for describing relativistic bound states in QFT, can be done simply and explicitly for a few models, such as the Yukawa model [6] and QED [9]. In this paper, we have extended the method to models, such as those 
mediated by non-Abelian gauge fields, for which a simple, explicit solution cannot be obtained. In such cases we show that the reduced Lagrangian and corresponding field equations can be constructed in an exact, but implicit, form in terms of functional equations.

In the case of scalar particle fields with scalar and/or vector coupling, the solutions are given semi-explicitly in terms of series, which follow from an integral operator fraction of the form $(1+\alpha\langle D * \ldots\rangle)^{-1}$. Two different operator series are obtained, applicable for strong and weak coupling, respectively. In the case of non-Abelian mediating gauge fields, the effective interaction is shown to have an expansion in odd powers of the coupling constant. Explicit expressions for the first few terms of this series are presented.

* A brief sketch of scientific interests and research activity of the untimely-deceased Dr. V. Shpytko is given in Ref. [11].
[1] E. E. Salpeter, H. A. Bethe, Phys. Rev. 84, 1232 (1951); H. A. Bethe, E. E. Salpeter, Quantum mechanics of Oneand Two-Electron Atoms (Springer Verlag, Berlin, 1957).

[2] E. Brezin, C. Itzykson, J. Zinn-Justin, Phys. Rev. D 1, 2349 (1970); I. T. Todorov, Phys. Rev. D 3, 2351 (1971); C. Fronsdal, R. W. Huff, Phys. Rev. D 3, 933 (1971).

[3] A. O. Barut, W. Rasmussen, J. Phys. B6, 1695 and 1713 (1973); A. O. Barut, in Groups, Systems and Many-Body Physics, P. Kramer, M. D. Cin, eds., (Braunschweig, Wiesbaden: Vieweg, 1980), p. 285.

[4] P. Havas, in Problems in the Foundations of Physics, M. Bunge, ed., (Springer, Berlin, 1971), p. 31; P. Ramond, Phys. Rev. D 7, 449 (1973).

[5] J. W. Darewych, Annales F. Louis de Broglie (Paris) 23, 15 (1998); J. W. Darewych, in Causality and Locality in Modern Physics Proceedings of a Symposium in honour of Jean-Pierre Vigier, G. Hunter, S. Jeffers, J. -P. Vigier, eds., (Kluwer Academic Publishers, Dor- drecht, 1998), p. 333.

[6] J. W. Darewych, Can. J. Phys. 76, 523 (1998); M. Barham, J. Darewych, J. Phys. A 31, 3481 (1998); B. Ding, J. Darewych, J. Phys. G 26, 907 (2000) and Nucl. Phys. B (Proc. Suppl.) 90, 136 (2000).

[7] J. Darewych, Condens. Matter Phys. 1, 593 (1998).

[8] V. Shpytko, J. Darewych, Phys. Rev. D 64, 045012 (2001)

[9] J. Darewych, A. Duviryak, Phys. Rev. A 66, 032102 (2002).

[10] H. C. von Baeyer, Phys. Rev. D 12, 3086 (1975).

[11] A. Duviryak, V. Tretyak, Yu. Yaremko, "Volodymyr Shpytko (1966-2001): The creative way", in Collected Physical Papers of Schevchenko Scientific Society, V. 4, Lviv, 2001, pp. 513-516 [А. Дувіряк, В. Третяк, Ю. Яремко, "Воло,димир Шпитко (1966-2001): творчий шлях", в Фізичний збірник HTIII, Т. 4, Львів, 2001, сс. 513$516]$.

\title{
РЕДУКОВАНІ ЛАГРАНЖІЯНИ ДЛЯ ПОЛЬОВИХ ТЕОРІЙ 3 НЕЛІНІЙНИМ ЗВ’ЯЗКОМ
}

\author{
В. Є. ІШпитко ${ }^{1}$, Ю. В. Даревич ${ }^{2}$ \\ ${ }^{1}$ Інститут фізики конденсованих систем НАН Украӥни, \\ вул. Сбениіиького, 1, Львіб, 79011, Украӥна, \\ ${ }^{2}$ Факультет фізики та астрономій, Йорксъкий університет, \\ Торонто, Онтаріо, МЗЈ 1РЗ, Канада
}

Вивчено переформулювання різних моделей квантової теорії поля, у якому польові рівняння використано для вираження полів - носіїв взаємодії в термінах полів матерії. Зокрема розглянуто скалярну KЕд, та SU(2)-калібрувальні поля. Показано, що для цих випадків члени взаємодії переформульованого лагранжіяна можна зобразити як ряд, члени якого містять лише поля матерії та функпії Іріна полів — носіїв взаємодії. 